\title{
Chaos and the continuum limit in charged particle beams
}

\author{
Henry E. Kandrup \\ Department of Astronomy, Department of Physics, and Institute for Fundamental Theory, University of Florida, \\ Gainesville, Florida 32611, USA \\ Ioannis V. Sideris \\ Department of Physics, Northern Illinois University, DeKalb, Illinois 60115, USA
}

Courtlandt L. Bohn*

Department of Physics, Northern Illinois University, DeKalb, Illinois 60115, USA

and Fermilab, Batavia, Illinois 60510, USA

(Received 7 March 2003; revised manuscript received 15 August 2003; published 14 January 2004)

\begin{abstract}
We investigate the validity of the Vlasov-Poisson equations for calculating properties of systems of $N$ charged particles governed by time-independent Hamiltonians. Through numerical experiments we verify that there is a smooth convergence toward a continuum limit as $N \rightarrow \infty$ and the particle charge $q \rightarrow 0$ such that the system charge $Q=q N$ remains fixed. However, in real systems $N$ and $q$ are always finite, and the assumption of the continuum limit must be questioned. We demonstrate that Langevin simulations can be used to assess the importance of discreteness effects, i.e., granularity, in systems for which the physical particle number $\mathcal{N}$ is too large to enable orbit integrations based on direct summation of interparticle forces. We then consider a beam bunch in thermal equilibrium and apply Langevin techniques to assess whether the continuum limit can be safely applied to this system. In the process we show, especially for systems supporting a sizable population of chaotic orbits that roam globally through phase space, that for the continuum limit to be valid, $\mathcal{N}$ must sometimes be surprisingly large. Otherwise the influence of granularity on particle orbits cannot be ignored.
\end{abstract}

DOI: 10.1103/PhysRevSTAB.7.014202

PACS numbers: 29.27.-a, 45.10.-b, 52.25.Fi

\section{INTRODUCTION}

A standard, often tacit, assumption in theoretical investigations of charged particle beams is that particle correlations are unimportant. With this assumption, one applies the Vlasov-Poisson equations to calculate the distribution function of the particles in the six-dimensional phase space of a single particle $[1,2]$. If the system is in static equilibrium, then the distribution function can be expressed as a function of isolating integrals of particle motion in the mean potential. For example, any function of the Hamiltonian derived from the mean space-charge potential is a solution of the Vlasov-Poisson equations, though not all such functions correspond to stable equilibria [3]. For systems not too far from equilibrium, one can invoke a perturbation theory using Vlasov-Poisson to calculate the evolution of the distribution function [4]. Such techniques are powerful and mature, but the underlying assumption must be questioned [5].

A major justification typically provided for using Vlasov-Poisson is the length of the collisional relaxation time $t_{R}$. By regarding this relaxation as the consequence of an incoherent sum of binary encounters [6], one finds $t_{R} \propto \overline{\boldsymbol{v}}^{3} /\left(q^{2} n \ln \Lambda\right)$, where $\overline{\boldsymbol{v}}$ is a typical speed associated with random motions, $q$ is the particle charge, $n$ is a characteristic number density, and $\ln \Lambda$ is the so-called

*Electronic address: clbohn@niu.edu
Coulomb logarithm, with $\Lambda$ scaling as a positive power of the number of constituent particles $N$. Upon assuming the bulk kinetic and potential energies are comparable in magnitude, this result becomes $t_{R} \sim 0.1(N / \ln N) t_{D}$, with $t_{D} \sim R / \bar{v}$ denoting a dynamical (orbital) time scale that depends on the system size $R$. Given plausible parameter values for real high-brightness beams, it becomes immediately clear that $t_{R} \gg t_{D}$. For example, let $\mathcal{N}$ denote the physical number of charges comprising a beam bunch, and consider $N=\mathcal{N}=6.25 \times 10^{9}(1 \mathrm{nC})$. This gives $t_{R} \sim 10^{7} t_{D}$, suggesting that collisions are unimportant to the evolution of beams in, for example, linear accelerators. However, consider simulations for which the number of macroparticles $N \ll \mathcal{N}$. The estimated $t_{R}$ may now be small enough to drive collisional relaxation during the simulation, in which case it is an artifact of the relatively small number $N$ of macroparticles.

As is evident in its derivation from the BogoliubovBorn-Green-Kirkwood-Yvon hierarchy [7], a VlasovPoisson system having total charge $Q$ represents a continuum limit in which the number of particles $N \rightarrow \infty$ while their individual charge $q \rightarrow 0$ such that $q N=Q$. By contrast, real systems contain a finite number of particles $\mathcal{N}$, and real charges have nonzero magnitudes. Vlasov-Poisson is thereby unrealistic, yet for the reasons stated, it is used to describe systems in the real world. The obvious question, therefore, is (1) to what extent do predictions derived from Vlasov-Poisson 
adequately describe real finite- $\mathcal{N}$ systems? A related question is (2) to what extent do predictions derived from a simulation involving $N<\mathcal{N}$ macroparticles adequately describe the real $\mathcal{N}$-body system? The present paper is concerned with how discreteness effects, i.e., granularity, influence the answers to both questions.

\section{PRELIMINARIES}

We adopt and extend techniques recently used for similar investigations of the gravitational $\mathrm{N}$-body problem [8,9]. That work concerned static potentials (analogous to those of Sec. III below) for which, in the continuum limit, the orbits are either all regular or essentially all chaotic, permitting one to isolate discreteness effects on both orbital types. Static beam potentials, however, will normally contain an admixture of both regular and chaotic orbits. Consider, for example, a beam bunch in thermal equilibrium [10], wherein space charge is important (e.g., that of Sec. IV below). The density in the central regions is quasiuniform, giving rise to nearly linear forces and thereby mostly regular orbits. In the outer regions the density falls to a diffuse tail over a few Debye lengths. The corresponding spacecharge force is thus nonlinear and, if the configuration lacks spherical symmetry, it may give rise to a sizable population of chaotic orbits that roam through global regions of phase space, i.e., "globally chaotic" orbits. The bulk potential then supports an admixture of orbital types, and finite- $N$ effects facilitate transitions between regularity and chaos. The question of how granularity influences these orbits is of interest, particularly in the context of the transition to the continuum limit.

\section{A. Frozen $N$-body models}

To facilitate our analysis, we formulate the VlasovPoisson equations in dimensionless variables. To model discreteness effects, we consider static systems and replicate their respective density profiles with distributions of $N$ identical macroparticles. These macroparticles reside at fixed coordinates that are randomly selected but weighted in keeping with the system's density profile, thereby resulting in a "frozen- $N$ representation." A charge $q$ is assigned to each macroparticle such that $q N=Q$, the total charge of the physical system. Our use of dimensionless variables makes the geometry of the system invariant with respect to the choice of $N$, thereby putting all frozen- $N$ representations and the Vlasov-Poisson system on the same footing. Of course, only one choice of $N$ is real, i.e., $N=\mathcal{N}$. However, real high-charge beam bunches can have a huge number of particles, making $\mathcal{N}$-body simulations impractical. One must therefore consider implications of simulations with $N<\mathcal{N}$; our dimensionless formulation provides the means to do so.

The total potentials associated with the dimensionless Vlasov-Poisson system and corresponding frozen- $N$ sys- tems give rise to dimensionless equations of test-charge motion. For a frozen- $N$ potential, the equation of motion implicitly equates the charge of the test particle to the charge of each macroparticle. Having constructed a frozen- $N$ system, we then compute orbits of test particles and determine to what extent the value of $N$ influences the statistical behavior of collections of test particles.

By freezing the positions of the $N$ macroparticles, we turn off any collective relaxation that may arise in a real system. However, we retain the collective space-charge force calculated from the static density distribution via Poisson's equation, as well as discreteness effects on testparticle orbits.

The use of frozen- $N$ systems drastically reduces the computational time, which in turn enables using much larger values of $N$ than would otherwise be possible. This allows us to demonstrate the validity of a Langevin/ Fokker-Planck description of collisional, i.e., "discreteness," effects on particle orbits in large- $N$ systems [11-13]. We then use this description to explore discreteness effects for particle numbers $N \rightarrow \mathcal{N}$ that are found in real high-charge beam bunches, i.e., values too large to permit even frozen- $N$ simulations. We are thereby equipped to answer questions (1) and (2) posed earlier. In the process, we demonstrate that discreteness effects can be critically important even within just a few dynamical times $t_{D}$, especially for potentials that support a sizable population of globally chaotic orbits. In such systems one must take care in using Vlasov-Poisson, especially when treating beams for which the detailed evolution and time scales are of practical concern. Such is often the case respecting, e.g., high-brightness, highaverage-current beams that require tight controls on emittance and/or beam halo [14].

\section{B. Methodology for orbital analysis}

We begin by outlining the methodology used to explore orbits in the potentials considered herein, which is essentially the same as applied in previous studies, e.g., [10]. We integrated the equation of motion for at least 100 orbital periods and, in most cases, for $\gtrsim 200$ orbital periods. The integrations were performed using a fifthorder Runge-Kutta algorithm [15] with variable time step. For each orbit, the fractional energy conservation was $<10^{-8}$ at each time step, and $<10^{-6}$ over the whole orbit. As the integration proceeded, we also computed the largest Lyapunov exponent for each orbit using a standard algorithm from chaotic dynamics [16]. The idea is to evolve two initial conditions that start with a very small separation for about one dynamical time, then renormalize to bring the two particles close together again, and repeat the process until the average exponent associated with the orbital separation converges to an almost stable value. Typically convergence was achieved within $\sim 100$ orbital periods. In general the Lyapunov exponent in the 
smooth potential, $\chi_{S}$, was much smaller than that in the corresponding frozen- $N$ potential, $\chi_{N}$.

After computing the orbits, we extracted the power spectrum for each orbit using a fast-Fourier-transform algorithm [15], and we then computed the "complexity" of the orbit [17]. This entailed determining for each orbit the quantities $n_{x}, n_{y}$, and $n_{z}$, defined as the minimum number of frequencies required to capture a fixed fraction $k$ of the power in each direction, and then assigning a total complexity $n(k)=n_{x}+n_{y}+n_{z}$. To obtain reasonably sharp Fourier spectra, we recorded data at least 100 times per orbital period. Then we sorted the spectral frequencies in descending order and, starting from the highest frequency, added as many frequencies as were needed to reach $90 \%$ of the total power, i.e., $k=0.9$. For reasons explained and justified elsewhere [10], we categorized an orbit as chaotic if $n(0.9)>20$.

Computations in the frozen- $N$ potentials involved exact direct-summation $N$-body integrations. Granularity in itself makes all test-particle orbits in the frozen- $N$ configurations chaotic [18], causing exponential divergence of nearby test-particle trajectories that persists until the separation between the trajectories becomes large compared to a typical intermacroparticle spacing. We verified that the statistics of the computed orbits, as probed by their Lyapunov exponents and complexities, were unchanged by choosing more stringent requirements for fractional energy conservation, e.g., $<10^{-10}$ for each time step and $<10^{-8}$ for the entire orbit. Moreover, differences between individual orbits in different frozen- $N$ potentials with the same $N$ were much larger than differences arising from different choices of computational accuracy. Integrating a single orbit in a $N=10^{6}$ potential typically took $\sim 4$ hours on a Pentium III computer.

\section{Modeling $N$-body orbits and flows by Gaussian white noise}

Conventional wisdom holds that effects from granularity reflect a Markov process that can be idealized as friction and Gaussian (nearly) white noise in the context of a Fokker-Planck description [11]. Taken literally, this suggests that individual $N$-body orbits can be well mimicked by Langevin simulations. However, it is unclear $a$ priori to what extent this is really true. The original derivation of the Fokker-Planck equation (and most if not all its tests) restricts attention to the statistical properties of collections of orbits, or to distribution functions, over time scales that are long enough to submit to a Markov treatment. It also carries an implicit assumption that the bulk potential in which the particles evolve is nonchaotic. An open question is whether the friction/ noise paradigm correctly describes shorter-time orbital behavior, especially in a chaotic potential.

If the Langevin description is valid, a simple rule connects $N$ to the strength of the friction. Assuming that the noise is characterized by a temperature per unit mass $\Theta$ comparable to the magnitude of the particle energy, the coefficient of dynamical friction $\eta$ defines a relaxation time $t_{R} \equiv \eta^{-1}$. However, per the Introduction, the binary-encounter approximation yields $t_{R} \propto$ $(N / \ln N) t_{D}$, and so $\eta$ should scale as $\eta \propto \ln N / N$.

To justify a Fokker-Planck description, one must demonstrate that frozen- $N$ simulations with specified $N$ can be well mimicked by Langevin simulations with $\eta \propto$ $\ln N / N$. Here two practical issues arise. The first is that the limited range of $N$ that can be explored makes testing the $\ln N$ dependence impractical: for $N<10^{3}$ or so, the very notion of a bulk potential fails; for $N>10^{6}$ computations become prohibitively expensive. One must instead be content with testing the scaling $\eta \propto N^{-1}$, i.e., $\ln \eta=$ $p-\ln N$ with $p$ being a constant. The second issue is more serious. The usual Langevin equation allows energy to vary on a time scale $t_{R}$, but test-particle energy is conserved absolutely in frozen- $N$ potentials. Energy conservation must be imposed on any scheme intended to mimic orbits in these potentials. For this reason, the noisy integrations described herein were performed using a modified energy-conserving noise previously devised for the study of gravitational frozen- $N$ potentials $[8,9]$.

\section{III. $N$-BODY ORBITS AND FLOWS IN REGULAR AND CHAOTIC POTENTIALS}

\section{A. Models considered}

Consider a uniform-density, ellipsoidal beam bunch with triaxial symmetry, axial dimensions $(a, b, c)$, and total charge $Q=1$ :

$$
\rho(\mathbf{r})=\frac{3}{4 \pi a b c} \times \begin{cases}1, & \text { if } R^{2} \leq 1, \\ 0, & \text { if } R^{2}>1,\end{cases}
$$

with $R^{2}=(x / a)^{2}+(y / b)^{2}+(z / c)^{2}$. This density generates a repulsive space-charge potential inside the beam of the form

$$
\Phi_{\mathrm{sc}}(\mathbf{r})=-\frac{1}{2}\left(\omega_{a}^{2} x^{2}+\omega_{b}^{2} y^{2}+\omega_{c}^{2} z^{2}\right)
$$

Suppose, however, that it is countered by an external confining potential $\Phi_{\text {ext }}=-2 \Phi_{\mathrm{sc}}$, and that there is a spherically symmetric spike of charge $\mathcal{Q}$ and size $\ell$ at the centroid. The total potential, in Gaussian units, is then

$$
\Phi(\mathbf{r})=\frac{1}{2}\left(\omega_{a}^{2} x^{2}+\omega_{b}^{2} y^{2}+\omega_{c}^{2} z^{2}\right)-\frac{\mathcal{Q}}{\sqrt{r^{2}+\ell^{2}}} .
$$

Taking $a=1.95, b=1.50$, and $c=1.05$ yields the frequencies $\omega_{a}=0.4663, \omega_{b}=0.5508$, and $\omega_{c}=0.6753$ [19], which implies a dynamical time $t_{D} \sim 2 \pi / \omega \sim 10$. We also take $\ell=10^{-3}$. The potential $\Phi$, though not representative of "real" beams (e.g., we do not let the charge distribution adjust to screen the central spike Q), has significant pedagogical value. Via the choice of $\mathcal{Q}$, it can be made to support only regular orbits or (almost) 
only globally chaotic orbits, thereby permitting a clean study of discreteness effects on both types of orbits.

Frozen- $N$ representations of the uniform ellipsoid having a charge density of the form

$$
\rho_{N}(\mathbf{r})=\frac{Q}{N} \sum_{i=1}^{N} \delta\left(\mathbf{r}-\mathbf{r}_{i}\right), \quad Q=1,
$$

were generated by randomly sampling the uniform density $\rho(\mathbf{r})$. The corresponding potential is

$$
\Phi_{N}(\mathbf{r})=\frac{1}{N} \sum_{i=1}^{N} \frac{1}{\sqrt{\left(\mathbf{r}-\mathbf{r}_{i}\right)^{2}+e^{2}}},
$$

wherein $e$ is a tiny softening parameter whose purpose is to avoid singularities that arise occasionally when the separation between two particles goes to zero [20]. Such events are rare, but when they occur, they slow down the computations considerably by necessitating extremely small time steps to retain sufficient accuracy. If $e$ is small enough that the probability of two orbits approaching within a distance comparable to $e$ is extremely small, then its precise value has no statistically significant impact on either the computed values of Lyapunov exponents or the Fourier spectra of orbits [8]. However, if $e$ is too large, there is an artificial reduction in the sizes of the computed Lyapunov exponents. Now, for a system of fixed size $R$, the typical interparticle separation decreases with increasing $N$, and progressively smaller values of $e$ are needed. We found empirically that for $N=10^{5.5}$, the orbital dynamics with $e=10^{-4}$ and $10^{-5}$ were statistically indistinguishable. The largest frozen- $N$ system considered herein has $N=10^{6}$, so we chose $e=10^{-5}$ for all of our experiments.

Choosing $\mathcal{Q}=0$ leads to what we shall call model 1 , an exact Vlasov-Poisson equilibrium [21]. Here, the net force inside the bunch is linear in the coordinates and the potential is manifestly integrable: all orbits are regular; all charges orbit with the same frequencies. Consider the evolution of an initially localized clump of test charges. In the absence of discreteness effects, the clump will experience no systematic phase mixing and no emittance growth. Hence, all emittance growth associated with a frozen- $N$ representation of this potential must be attributed to discreteness effects. By contrast, suppose $Q=$ $10^{-1.5} Q=0.03162$ and $\ell=10^{-3}$, leading to what we shall call model 2. Then, for test particles that are restricted energetically to lie inside the ellipsoidal boundary, the phase space is almost completely chaotic, and almost all test-particle orbits are globally chaotic.

The potential of Eq. (3) is analogous to a gravitational potential constituting a simple model of an elliptical galaxy with a supermassive black hole at its centroid. In that context the potential and its frozen- $N$ representation have been analyzed previously $[8,22]$. Herein we augment the earlier results for a more complete visualization of the approach to the continuum limit as $N$ is increased.

\section{B. Phase mixing and the continuum limit}

To explore the influence of discreteness effects on phase mixing, we generated clumps of 1600 localized initial conditions sampling a phase-space region of size $|\Delta \mathbf{r}| \sim$ $|\Delta \mathbf{v}| \sim 10^{-3}$ and monitored their evolution in both the smooth potential and the corresponding frozen- $N$ potential. A convenient diagnostic for visualizing and quantifying the impact of discreteness effects is the clump emittance, which we define as

$$
\epsilon=\left(\epsilon_{x} \epsilon_{y} \epsilon_{z}\right)^{1 / 3}
$$

with

$$
\epsilon_{i} \equiv \sqrt{\left\langle r_{i}^{2}\right\rangle\left\langle v_{i}^{2}\right\rangle-\left\langle r_{i} v_{i}\right\rangle^{2}}, \quad(i=x, y, z)
$$

Clumps evolved in the smooth, integrable potential of model 1 exhibit no systematic tendency to disperse. Because each orbit is harmonic, the charges remain close together, returning to their original $x_{0}, y_{0}, z_{0}$ after periods $\tau_{x}, \tau_{y}, \tau_{z}$, respectively. Granularity in the frozen- $N$ potential breaks this periodicity and triggers a systematic spread. The situation is very different for model 2 where, even in the continuum limit, the phase space is almost completely chaotic. The clumps phase mix exponentially fast in the smooth potential; incorporating granularity only accelerates the process. Even for the smooth potential, a time $t \sim 10 t_{D}$ is sufficient for particles to sample most of their energetically accessible phase space.

Plots of clump emittance in Figs. 1 and 2 enable one to contrast regular phase mixing in model 1 with chaotic phase mixing in model 2 . In model 1 it is unclear for sufficiently small $N$ [cf. Fig. 1(a)] whether $\epsilon(t)$ grows exponentially or as a power law. However, for $N \geq$ $10^{3.5}$, the growth is distinctly slower than exponential. Indeed, the data for $N \geq 10^{3.5}$ are well fit by a power law $\epsilon \propto(t / N)^{1 / 2}$. By extrapolating to the continuum limit, one uncovers the expected result that there is no systematic emittance growth in the smooth potential.

By contrast, in the smooth albeit chaotic potential of model 2 [cf. Fig. 2(i)], the emittance should grow exponentially as initially nearby orbits diverge. Indeed, during the interval $10<t<100, \epsilon(t)$ exhibits a roughly exponential growth at a rate comparable to the value of a typical Lyapunov exponent $\chi_{S}$ for the orbits. Discreteness effects clearly accelerate chaotic mixing. For smaller particle number, $N=10^{2.5}$ and $10^{3.5}$, the clump grows exponentially at a rate $\gg \chi_{S}$, but for $N=$ $10^{4.5}$ and $10^{5.5}$ the evolution is more complicated. For these two cases the evolution decomposes into two largely distinct exponential phases, the former with growth rate $\gg \chi_{S}$ acting over short length scales, and the latter with growth rate $\sim \chi_{S}$ acting globally. For sufficiently small $N$, 

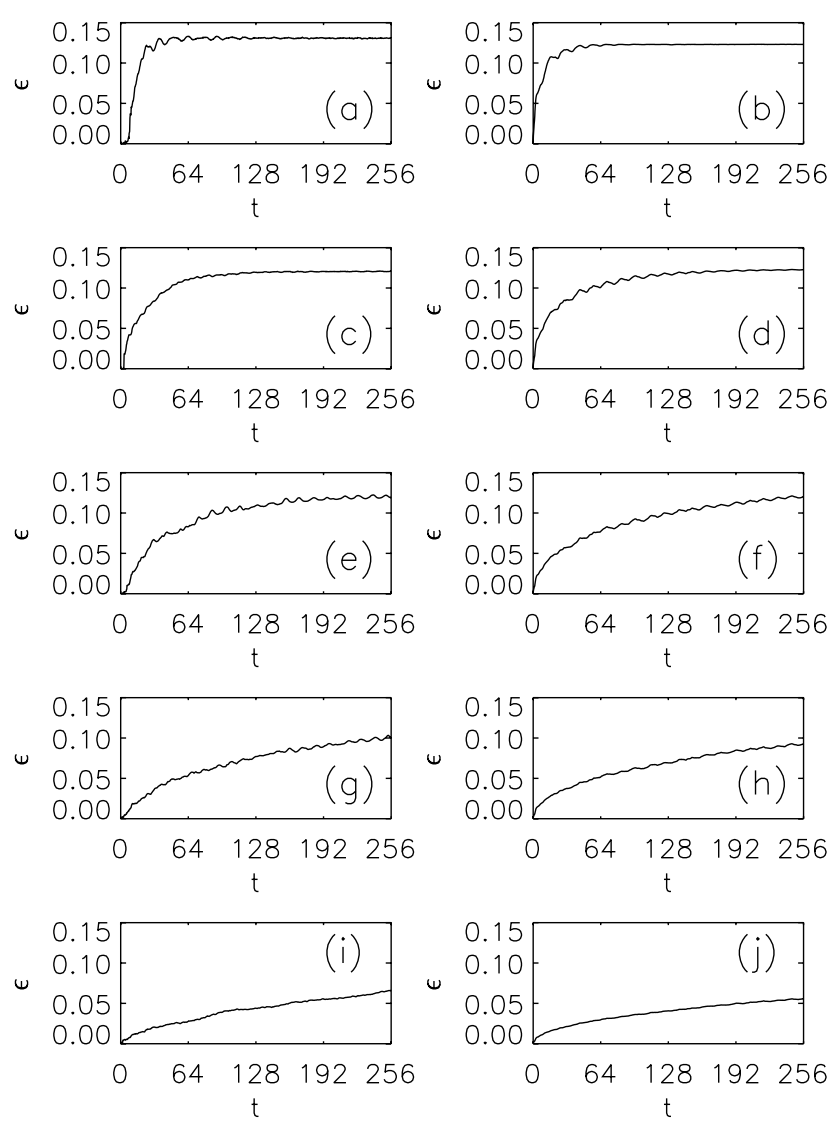

FIG. 1. Emittance $\epsilon=\left(\epsilon_{x} \epsilon_{y} \epsilon_{z}\right)^{1 / 3}$ vs time $t$ of a clump in model 1 wherein the total energy of each particle is $E=1.0$. The left and right columns correspond to the frozen- $N$ potential and energy-conserving white noise, respectively. (a) $N=10^{3.0}$. (b) $\eta=10^{-2.5}$. (c) $N=10^{3.5}$. (d) $\eta=10^{-3.0}$. (e) $N=10^{4.0}$. (f) $\eta=10^{-3.5}$. (g) $N=10^{4.5}$. (h) $\eta=10^{-4.0}$. (i) $N=10^{5.0}$. (j) $\eta=10^{-4.5}$.

discreteness effects dominate the dynamics, and in Figs. 2(a) and 2(c) the second exponential phase is nearly absent. For larger $N$, as in Figs. 2(e) and 2(g), the second exponential phase becomes more apparent.

The data of Figs. 1 and 2 also show that there are differences between the clump emittances in the frozen- $N$ and Langevin representations when $N$ is small, but that as $N$ increases, the clump emittances become increasingly comparable. Indeed, the data reflect the scaling relation $\ln \eta=p-\ln N$ with $p=0.5 \pm 0.1$. Several implications of crucial importance immediately arise such as the following:

(1) For sufficiently large $N$ the Langevin representation provides a good model of the orbital dynamics in the frozen- $N$ system. Thus the Langevin formalism seems useful for experiments with much larger values of $N$. By comparing Langevin results to those of the smooth potential, one can then identify, e.g., whether the continuum limit is justified for a real system with $N=\mathcal{N}$ particles.
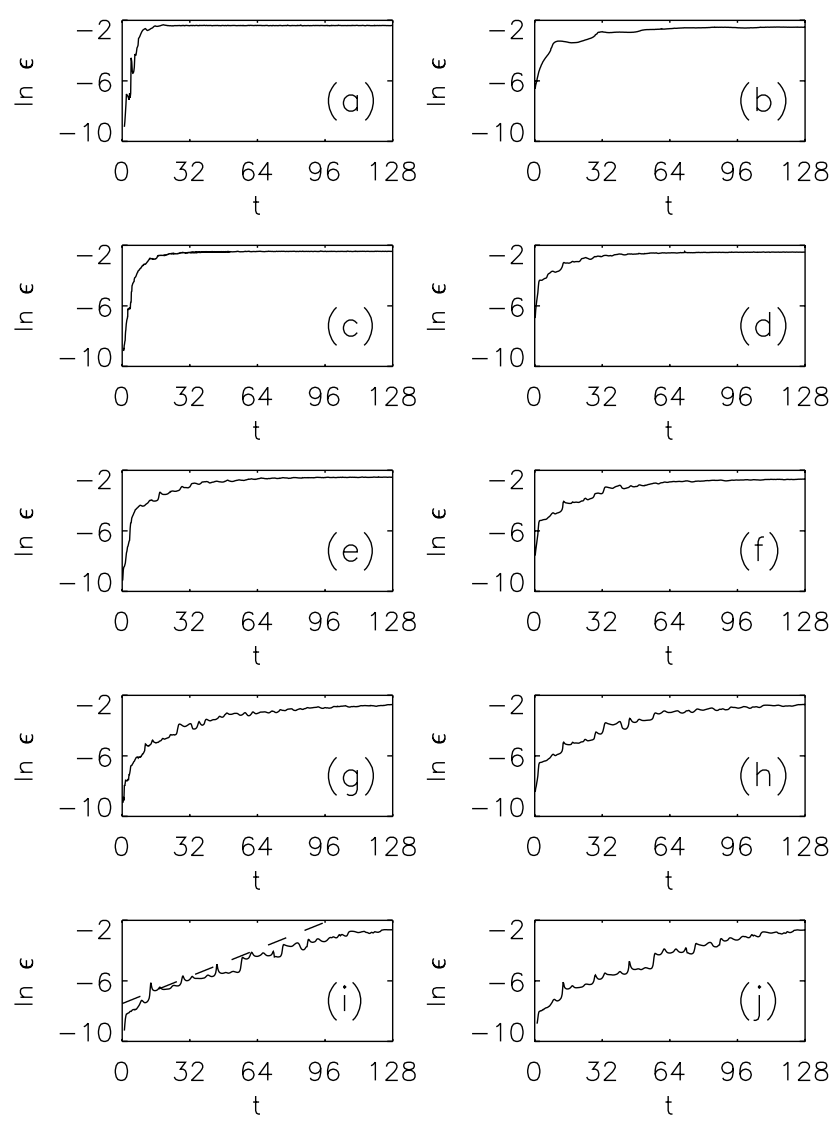

FIG. 2. Same as Fig. 1, but for model 2. Note the logarithmic scale. (a) $N=10^{2.5}$. (b) $\eta=10^{-2.0}$. (c) $N=10^{3.5}$. (d) $\eta=$ $10^{-3.0}$. (e) $N=10^{4.5}$. (f) $\eta=10^{-4.0}$. (g) $N=10^{5.5}$. (h) $\eta=$ $10^{-5.0}$. (i) The smooth potential. The dashed line has slope equal to the mean Lyapunov exponent $\left\langle\chi_{S}\right\rangle$ for the orbits. (j) $\eta=10^{-7.5}$, the largest noise amplitude that does not appreciably alter the results generated in the smooth potential.

(2) The Langevin results in Fig. 2(j), for which $\eta=$ $10^{-7.5}$, correspond to the largest noise amplitude that does not appreciably alter the results generated in the smooth potential. Thus, presuming the scaling of $\eta$ with $N$ remains unchanged for very large $N$; for model 2 one requires $N \gtrsim 10^{7}$ to suppress discreteness effects. In the context of macroparticle simulations, this is a large number indeed. In turn, were the corresponding physical system to have $\mathcal{N} \gtrsim 10^{7}$, one could hope the VlasovPoisson prescription would likewise strictly apply. However, some uncertainty must remain because frozen- $N$ models cannot include effects of multiparticle correlations such as particle-wave and wave-wave interactions that accompany charge redistribution [23].

(3) The collisional relaxation time $t_{R} \sim 0.1(N / \ln N) t_{D}$, yet discreteness effects can be important in a system with $N \sim 10^{6.5}$, even over times as short as $\sim 10 t_{D}$.

(4) Contrary to conventional wisdom, the $\mathrm{N}$-body Lyapunov exponent $\chi_{N}$ does not provide a useful measure of chaos, at least when chaos is viewed macroscopically. 
Instead, in the chaotic potential of model 2, the clump emittance scales as

$$
\epsilon(t) \propto(t / N)^{1 / 2} \exp \left(\chi_{S} t\right) .
$$

Granted that Gaussian white noise can mimic discreteness effects, the scaling of clump emittance with $N$ and $t$ is easily understood. At least for a harmonic potential, it is straightforward to derive analytic solutions to the Langevin equation for moments like $\left\langle x^{2}\right\rangle$ or $\left\langle x v_{x}\right\rangle$. Then, if the Langevin forces are small compared to all other forces relevant to the dynamics of the system, the Fokker-Planck equation for the clump emittance is [24]

$$
\frac{d \epsilon_{x}^{2}}{d t}=2 D\left\langle x^{2}\right\rangle-2 \eta\left(\left\langle x^{2}\right\rangle\left\langle v^{2}\right\rangle-\langle x v\rangle^{2}\right),
$$

in which the diffusion coefficient $D=\Theta \eta$, with $\Theta$ denoting the temperature per unit mass. If the initial emittance of the clump is extremely small, at early times $\left\langle x^{2}\right\rangle\left\langle v^{2}\right\rangle \approx\langle x v\rangle^{2}$. Averaging over oscillations gives $\left\langle x^{2}\right\rangle=$ $E / \omega^{2}$, with $E$ the initial energy. Accordingly, for early times, $\epsilon_{x} \simeq\left(2 D E t / \omega^{2}\right)^{1 / 2}$. Combining this result and the analogous formulas for $\epsilon_{y}$ and $\epsilon_{z}$ with the scaling relation $D \propto \eta \propto 1 / N$ leads immediately to $\epsilon \propto(t / N)^{1 / 2}$. After discreteness effects "kick" two nearly coincident orbits apart, they tend to diverge at a rate set by the Lyapunov exponent $\chi_{S}$ associated with the bulk potential. Equation (8) thus makes sense.

To reiterate, the results derived here for model 2 are likely generic for bulk density distributions corresponding to chaotic potentials. However, the results for model 1 are special in that there is zero systematic emittance growth in the continuum limit. If, as would be expected in real beams, the bulk potential exhibits at least some anharmonicities, regular phase mixing will cause clump emittances to grow linearly even in the continuum limit. Allowing for discreteness effects will again accelerate emittance growth; the exact form of this growth can be complicated, but will remain weaker than exponential.

\section{Individual orbits and the continuum limit}

The preceding results do not necessarily imply that individual orbits also converge toward characteristics in the smooth potential. To what extent, then, is it true that, as $N$ increases, individual trajectories converge towards their smooth-potential counterparts? The most compelling check is visual. As a simple, and extreme, example, set in Eq. (3) $a=b=c=1$, corresponding to $\omega_{a}=$ $\omega_{b}=\omega_{c}=1$, and $\mathcal{Q}=0$ to obtain a uniform-density spherical system. Select an initial condition that corresponds to a circular orbit and integrate it in a frozen- $N$ representation of the potential. Results appear in Fig. 3 for $10^{2.5} \leq N \leq 10^{5.5}$, with the smooth-potential (strictly circular) orbit plotted for comparison. For the smallest values of $N$ the orbit is nowhere near circular, nor is there even a sense of net circulation. As $N$ is increased,
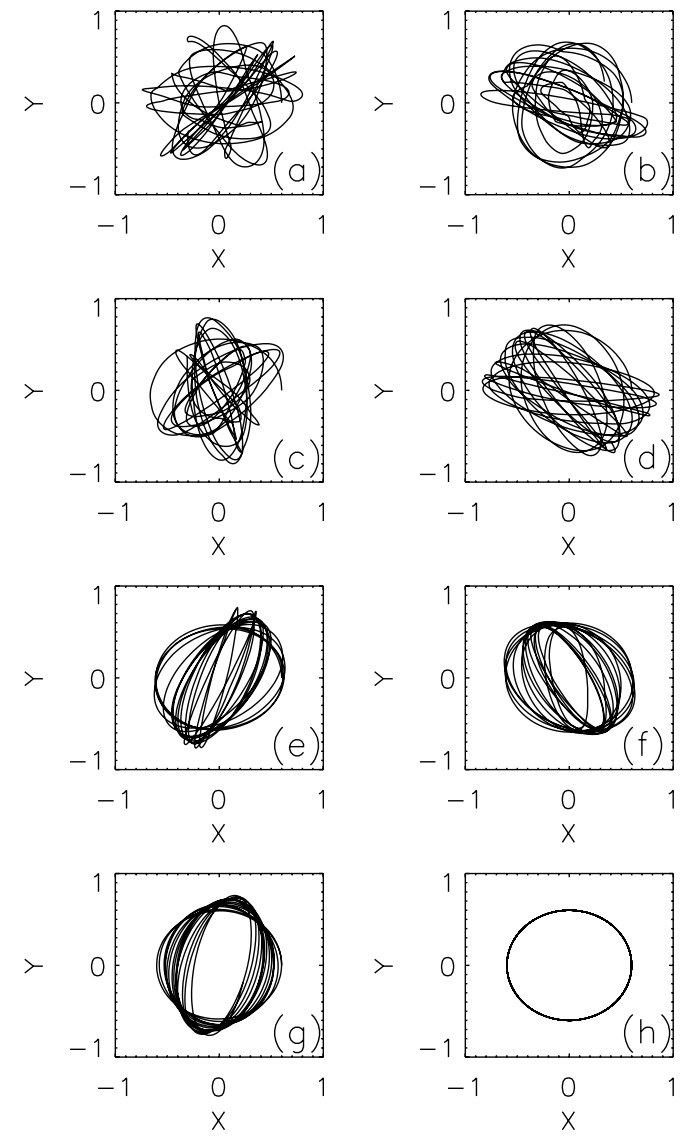

FIG. 3. The $(x, y)$ projection of a frozen- $N$ orbit generated from an initial condition $\left(x=0.6, y=z=0, v_{y}=0.6, v_{x}=\right.$ $v_{z}=0$ ) corresponding in the smooth spherical potential to a circular orbit. (a) $N=10^{2.5}$. (b) $N=10^{3.0}$. (c) $N=10^{3.5}$. (d) $N=10^{4.0}$. (e) $N=10^{4.5}$. (f) $N=10^{5.0}$. (g) $N=10^{5.5}$. (h) The smooth-potential orbit.

however, the orbit becomes "less tangled." For $N=10^{4.5}$ a clear sense of net circulation arises; for $N=10^{5.0}$ the orbit is clearly "centrophobic" (thus suggesting that angular momentum is at least approximately conserved); and for $N=10^{5.5}$ the orbit arguably resembles a "distorted" or precessing circular orbit.

Fourier spectra of the orbital data, examples of which appear in Fig. 4, corroborate the visual impression. For the three smallest values of $N,|x(\omega)|$ is obviously broadband, although there is a peak at or near the circular frequency $(\omega=1)$ associated with the smooth-potential orbit. For $N=10^{4.0}$ and $10^{4.5}$ the peak becomes appreciably sharper, and for $N=10^{5.0}$ and $10^{5.5}$ one sees only slight irregularities in the spectra which translate into the visual appearance of precession. The conclusion is obvious: as $N$ increases, frozen- $N$ orbits come to more closely approximate the smooth orbit, both visually and in terms of their power spectra. Analogous results obtain for more generic initial conditions evolved in this and other integrable potentials, such as the ellipsoidal potential of model 1 [8]. 

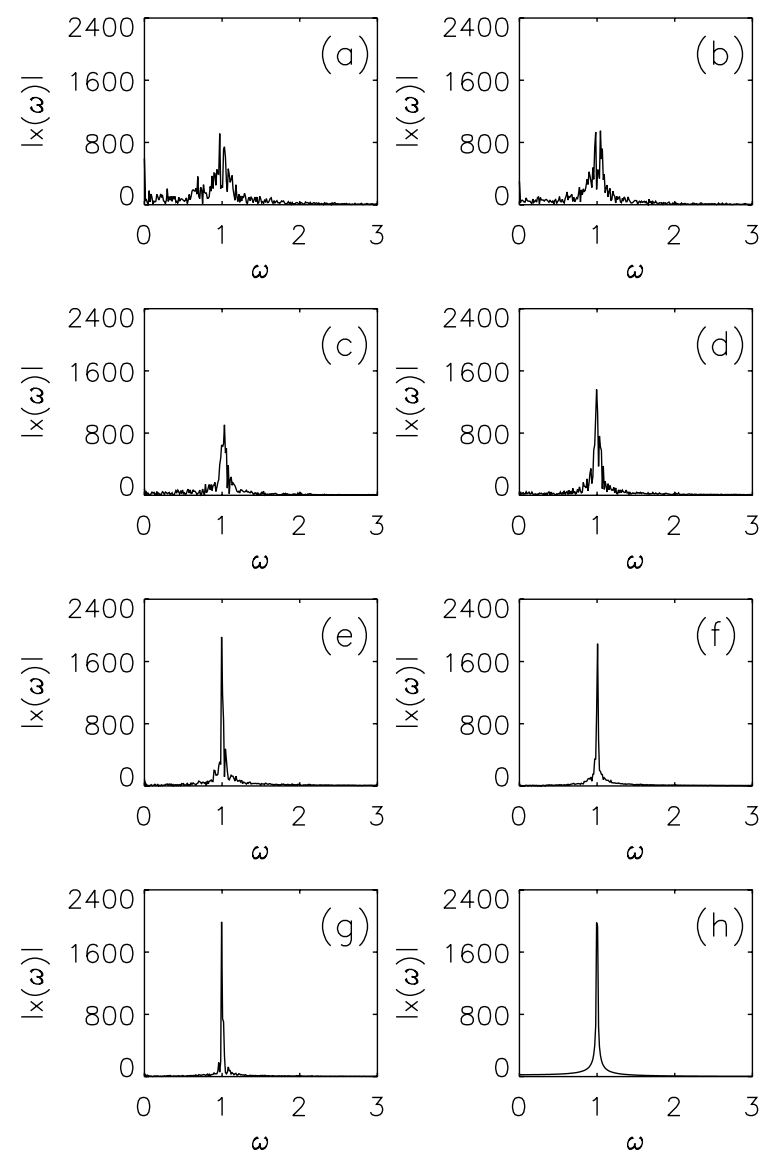

FIG. 4. The power spectrum $|x(\omega)|$ for the orbits exhibited in Fig. 3. (a) $N=10^{2.5}$. (b) $N=10^{3.0}$. (c) $N=10^{3.5}$. (d) $N=$ $10^{4.0}$. (e) $N=10^{4.5}$. (f) $N=10^{5.0}$. (g) $N=10^{5.5}$. (h) $\mathrm{The}$ smooth-potential orbit.

Convergence of Fourier spectra is of general importance for certain applications of nonlinear dynamics to many-body systems interacting via long-range forces. Many physical phenomena, including accelerator modes [25], modulational diffusion [26], and resonant relaxation [27], are attributed to resonant couplings between, e.g., natural frequencies of individual regular orbits and frequencies associated with time-dependent perturbations. However, such attributions can only be justified if the real $\mathcal{N}$-body orbits have frequency distributions that adequately approximate the frequencies associated with orbits in the smooth potential.

\section{IV. $N$-BODY ORBITS AND FLOWS IN A THERMAL-EQUILIBRIUM CONFIGURATION}

\section{A. The model}

We now consider a more realistic density-potential pair, that of a system of charges in thermal equilibrium. This system is of practical interest both for beam physics $[2,28,29]$ and nonneutral-plasma physics [1]. It consists of $\mathcal{N}$ identical charged particles, interacting electrostatically, that are constrained by linear restoring forces to manifest triaxial symmetry, the focusing forces in differ- ent orthogonal directions being characterized by unequal frequencies. The specific model we consider is one that, in the continuum limit, admits large measures of both regular and chaotic orbits, the latter reaching into the Debye tail where the net force is nonlinear. The model is constructed and its orbits are categorized elsewhere [10]. Upon defining a Debye length and plasma frequency in terms of the centroid number density $n(0)$, and then expressing length and frequency in units of these quantities, respectively, we express the corresponding number density normalized to $n(0)$ as

$$
n(\mathbf{r})=\exp \left[-\frac{1}{2} \Omega^{2} R^{2}-\Phi(\mathbf{r})\right],
$$

where $\Phi(\mathbf{r})$ is the space-charge potential that follows selfconsistently from Poisson's equation:

$$
\nabla^{2} \Phi(\mathbf{r})=-n(\mathbf{r}), \quad \Phi(0)=\nabla \Phi(0)=0 .
$$

Here $R^{2}=(x / a)^{2}+y^{2}+(z / c)^{2}$, with $a$ and $c$ being scale lengths, and $\Omega$ is a focusing strength. In general one cannot solve Eqs. (10) and (11) analytically. This makes the computation of orbits in the smooth potential much more difficult; however, these difficulties were overcome using numerical techniques described in [10].

Frozen- $N$ representations of the form

$$
n_{N}(\mathbf{r})=\sum_{i=1}^{N} \delta\left(\mathbf{r}-\mathbf{r}_{i}\right)
$$

were generated by randomly sampling the smooth density $n(\mathbf{r})$. The frozen- $N$ space-charge potential is given by

$$
\Phi_{N}(\mathbf{r})=\frac{1}{4 \pi} \frac{N}{\mathcal{V}} \sum_{i=1}^{N} \frac{1}{\sqrt{\left|\mathbf{r}-\mathbf{r}_{i}\right|^{2}+e^{2}}},
$$

with $N$ denoting the number of frozen particles, and

$$
\mathcal{V} \equiv \int_{V} d \mathbf{r} \exp \left[-\frac{1}{2} \Omega^{2} R^{2}-\Phi(\mathbf{r})\right]
$$

where the volume $V$ spans all of space. In practice one cannot precisely evaluate $\mathcal{V}$, even numerically, since $\Phi(\mathbf{r})$ can only be determined over a finite-size grid. We found $\mathcal{V}$ by using integration limits coinciding with the grid boundaries, and then renormalized by a small constant that made the plots of potential and density in the smooth versus frozen- $N$ configurations overlap perfectly.

The system considered here has parameters $a^{2}=0.5$, $c^{2}=1.5$, and $\Omega=1.0001 / \sqrt{3}$; we shall call this model 3 . They yield a dynamical time $t_{D} \sim 20$, which corresponds to the space-charge-depressed period, and define a beam carrying moderate space charge. Consider, for example, a proton bunch with temperature $k_{B} T=5 \mathrm{eV}$ spanning $3 \mathrm{~cm}$ full "radius" ( $\mathrm{rms}$ beam size $\tilde{y} \sim 1.3 \mathrm{~cm}$ ). The rms normalized emittance is $\tilde{y}\left[k_{B} T /\left(m c^{2}\right)\right]^{1 / 2} \simeq 1 \mu \mathrm{m}$, the Debye length is $\lambda_{D} \sim 2 \mathrm{~mm}$, the central density (in SI units) is $n(0)=\varepsilon_{0} k_{B} T /\left(e \lambda_{D}\right)^{2} \simeq 7 \times 10^{13} \mathrm{~m}^{-3}$, and the bunch charge is $Q=e n(0) \lambda_{D}^{3} \mathcal{V} \simeq 0.5 \mathrm{nC}$, i.e., $\mathcal{N} \simeq$ $3 \times 10^{9}$ protons. The tune depression is roughly 0.35 [28]. 


\section{B. Phase mixing of regular orbits}

We first discuss an initially localized clump of 1600 test charges evolved in frozen- $N$ representations of model 3, with total energy sufficiently small that the constant-energy hypersurface in the smooth potential is completely regular. Given how model 3 was made dimensionless, the size of the beam is $\sim 10$ units as opposed to $\sim 1$ unit in the case of models 1 and 2 , so the initial conditions of particles in the clump sampled a region 10 times larger: $|\Delta \mathbf{r}| \sim|\Delta \mathbf{v}| \sim 10^{-2}$. The emittance $\epsilon(t)$ for various choices of $N$ appears in the left-hand panels of Fig. 5. As for model 1 in Sec. III, the emittance scales as a power law rather than exponentially, and at least for $N=$ $10^{5.5}$ and $N=10^{6.0}$, it is well fit by $\epsilon \propto t^{1 / 2}$. The emittance $\epsilon(t)$ in the smooth potential is shown in Fig. 5(i). Here the evolution is clearly linear, not square root, which is the expected behavior for phase mixing in generic integrable potentials where nearby initial conditions correspond to slightly different orbital frequencies. We did not see this in the smooth potential of model 1 because there was no frequency spread. That the clump emittance grows much faster with $N=10^{6.0}$ than in the smooth potential shows clearly $10^{6}$ particles are insufficient to represent the smooth potential of model 3, even over an interval as short as $t=25 t_{D}$ (for comparison, a $1 \mathrm{GeV}$ proton linac would span roughly $40 t_{D}$ ).

The right-hand panels of Fig. 5 demonstrate that discreteness effects can again be well mimicked by energyconserving Gaussian white noise with $\ln \eta=p-\ln N$. For model 3, however, $p=1.5 \pm 0.2$, which differs from models 1 and 2 for which $p=0.5 \pm 0.1$. The emittance evolved with $\eta=10^{-6.5}$, the largest noise that does not significantly alter emittance growth in the smooth potential, is shown in Fig. 5(j). Presuming the scaling extends to larger $N$ and smaller $\eta$, the smallest number of macroparticles needed to replicate the behavior of regular orbits in the continuum limit of model 3 is $N \sim 10^{8}$.

\section{Phase mixing of chaotic orbits}

Next we discuss an initially localized clump of 1600 particles with total energy high enough to reach the Debye tail and make them globally chaotic in the smooth potential. The results appear in Fig. 6. As for model 2 in Sec. III, the evolution is exponential rather than power law, and granularity again has an important effect. Discreteness effects can still be well mimicked by noise with $\ln \eta=p-\ln N$ and $p=1.5$. Most striking, however, is the fact that even much weaker noise can now accelerate emittance growth appreciably. For these chaotic orbits, discreteness effects must correspond to a noise amplitude satisfying $\eta \leqq 10^{-8.0}$ before a continuum limit can be justified. Chaotic orbits are far more susceptible to low-amplitude noise than are regular orbits. Presuming again that the scaling holds, it follows that, for the case of chaotic orbits, the continuum limit cannot be justified for
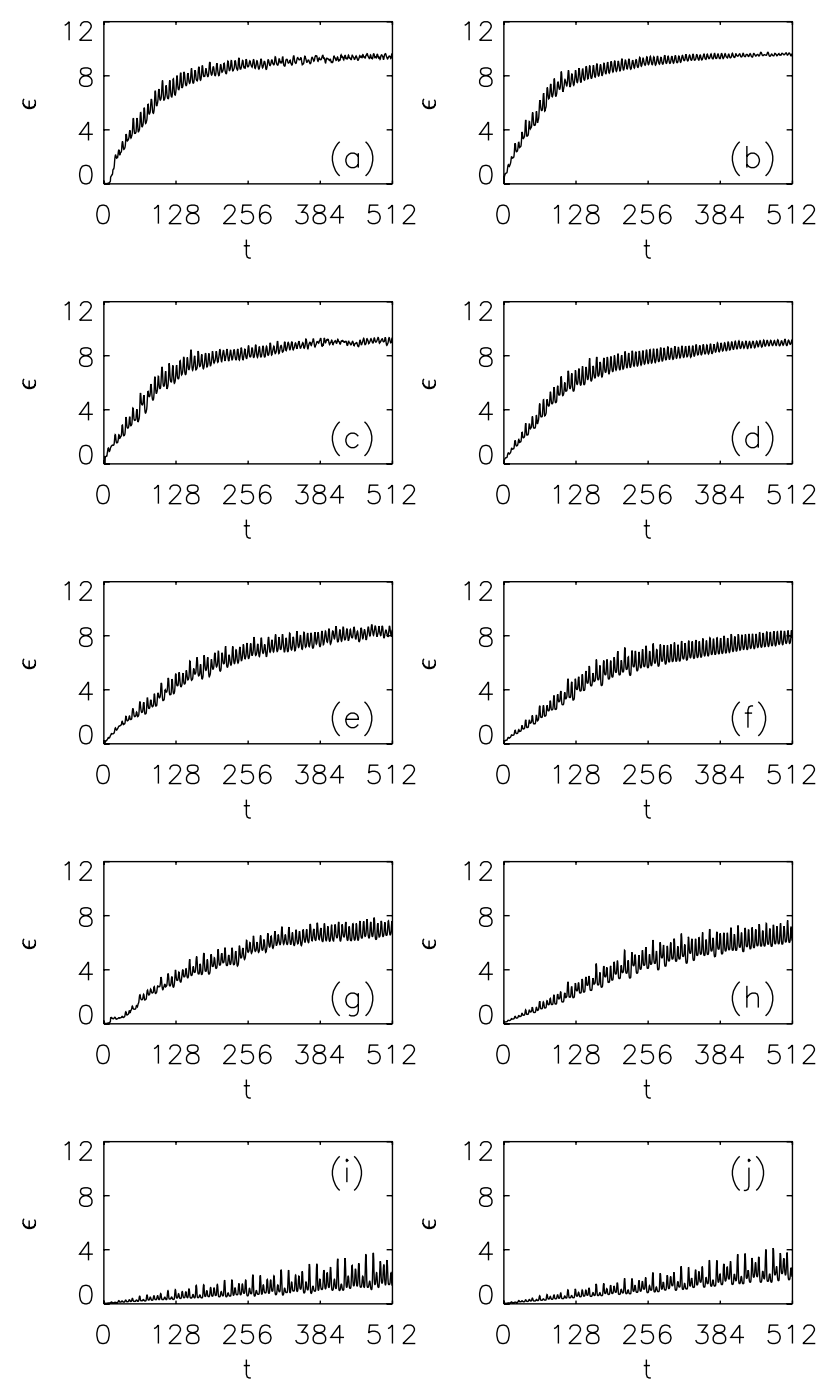

FIG. 5. Emittance $\epsilon=\left(\epsilon_{x} \epsilon_{y} \epsilon_{z}\right)^{1 / 3}$ vs time $t$ computed for a clump of regular orbits in model 3 , allowing for both frozen- $N$ backgrounds and energy-conserving white noise. (a) $N=10^{4.5}$. (b) $\eta=10^{-3.0}$. (c) $N=10^{5.0}$. (d) $\eta=10^{-3.5}$. (e) $N=10^{5.5}$. (f) $\eta=10^{-4.0}$. (g) $N=10^{6.0}$. (h) $\eta=10^{-4.5}$. (i) Unperturbed motion in the smooth potential. (j) $\eta=10^{-6.5}$, the largest value of $\eta$ that does not significantly impact emittance growth.

$N \lesssim 10^{9.5}$ over time scales $t \geqslant 25 t_{D}$. This means the Vlasov-Poisson equations are at best only marginally applicable to the real beam for which $N=\mathcal{N} \simeq$ $3 \times 10^{9}$. Accordingly, in studying the dynamics of beams with moderate space charge, one may not be able to assume with complete confidence that the continuum limit, and hence the Vlasov-Poisson prescription, is justified physically, even if the system is in a state of static equilibrium.

\section{Transitions between regular and chaotic behavior}

Model 3 differs from models 1 and 2 in that the smooth potential supports both regular and chaotic orbits. At low 

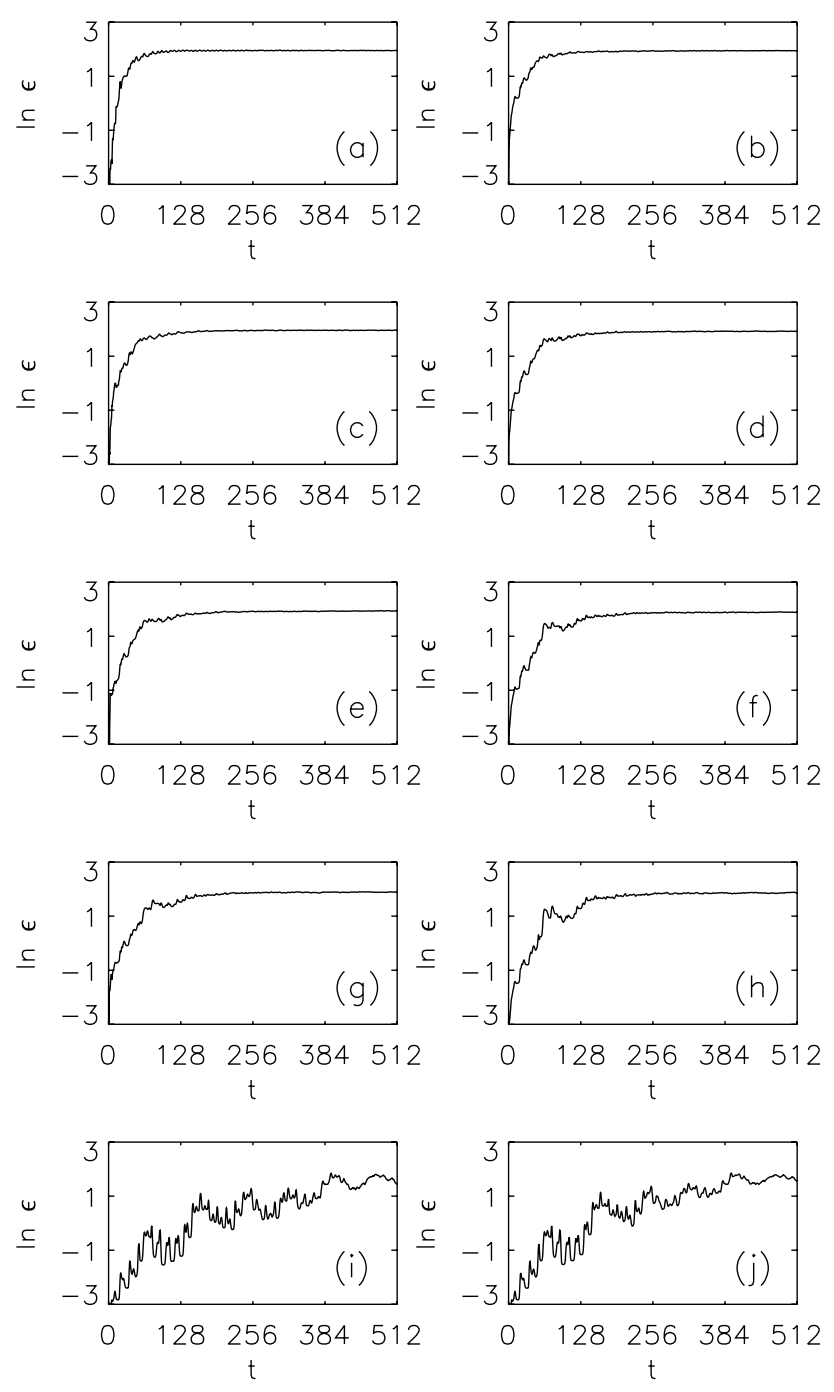

FIG. 6. Same as Fig. 5 but for a clump of chaotic orbits. (a) $N=10^{4.5}$. (b) $\eta=10^{-3.0}$. (c) $N=10^{5.0}$. (d) $\eta=10^{-3.5}$. (e) $N=10^{5.5}$. (f) $\eta=10^{-4.0}$. (g) $N=10^{6.0}$. (h) $\eta=10^{-4.5}$. (i) Unperturbed motion in the smooth potential. (j) $\eta=10^{-8.0}$, the largest noise amplitude that does not appreciably alter the results generated in the smooth potential.

energies, where the total potential is nearly harmonic, all orbits are regular, and discreteness effects can deflect only frozen- $N$ orbits from one regular trajectory to another. At higher energies, however, there is a complex coexistence of regular and chaotic orbits. Discreteness effects can now deflect orbits from regular to chaotic trajectories and vice versa [30]. Of obvious interest then is how fast such transitions occur. For example, an accelerator designer relying on the Vlasov-Poisson prescription and its inherent smooth potential would neglect these microscopic transitions. Thus their influence on phase mixing and its attendant emittance growth would be improperly modeled, and the computed results, e.g., evolutionary time scales and length scales, would be suspect. The consequences of such an omission depend on the problem at hand, but one might expect them to be most pronounced for nonequilibrium beams wherein chaotic dynamics is likely to be more prevalent [31].

Suppose we select an initially localized clump of particles all of which follow regular orbits in the smooth potential. Suppose further that all of these particles have energy sufficiently high that many other orbits with the same energy are chaotic. We now place this clump in a frozen- $N$ representation and integrate the orbits. The transition of an orbit from regular to chaotic (or, more precisely, from locally chaotic to globally chaotic) can be easily detected because the orbit suddenly becomes more "irregular" in appearance. If, moreover, large numbers of such transitions occur over very short times, they will make the clump emittance transition from power-law growth to roughly exponential growth.

To determine the relative fraction of the orbits that remain regular after some time $t$, we first recorded the phase-space coordinates of individual frozen- $N$ orbits at various times $t>0$. Then, using these coordinates as initial conditions, we evolved these orbits in the smooth potential to determine whether the resulting trajectories were still regular [complexity $n(0.9) \leq 20$ ] or whether instead they had become chaotic $[n(0.9)>20]$. Presuming the system is ergodic and discreteness effects are strong enough to convert any orbit from regular to chaotic (and vice versa), it would seem clear what the analysis ought to reveal. (1) At sufficiently long times, independent of $N$, the relative measure of chaotic orbits generated from any clump should (to within statistical uncertainties) coincide with the relative measure of chaotic orbits on the constant-energy hypersurface. (2) Assuming, however, that discreteness effects are more important for smaller $N$, the time required to converge asymptotically toward this value should be an increasing function of $N$, i.e., as $N$ increases, transitions should become more rare. As Fig. 7 shows, these expectations were in fact confirmed. For $N$ as small as $10^{4.5}$, about $35 \%$ of the orbits in a clump of initially regular orbits had become chaotic within a time $t=64$, only $\sim 3 t_{D}$, and the relative measure $f$ of chaotic orbits appears to have converged asymptotically toward a time-independent value $f \simeq 40 \%$ by $t=128$. For computations with $N=10^{5.0}, f$ grew more slowly in time, but by $t=512$ it had again approached a value $f \simeq 40 \%$. For larger values of $N, f$ remained a monotonically increasing function of time, but transitions were sufficiently rare that it did not reach a static value prior to $t=512 \simeq 25 t_{D}$.

\section{DISCUSSION AND CONCLUSIONS}

Viewed macroscopically, there is a precise sense in which, as the number of macroparticles $N$ increases, trajectories in frozen- $N$ systems converge toward their counterparts in the corresponding smooth potential. For very small $N,<10^{4}$ or so, the notion of an average bulk 


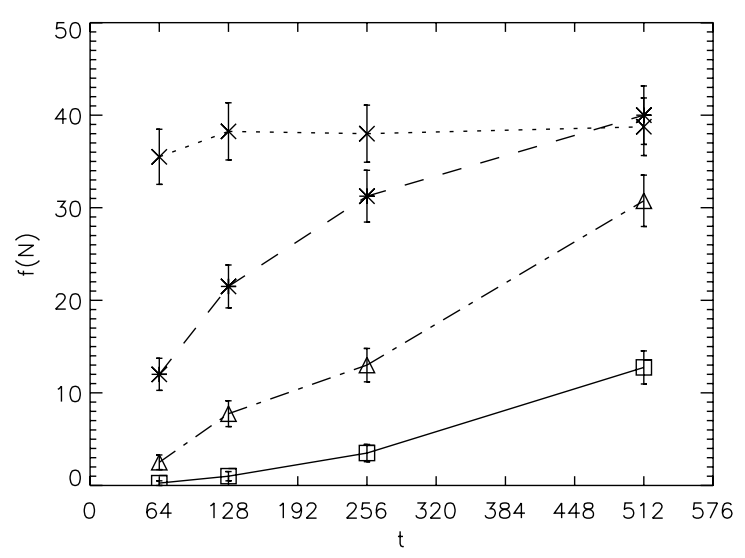

FIG. 7. The net percentage $f$ of orbits generated from a clump of regular initial conditions in a frozen- $N$ representation of model 3 that, as of time $t$, have been converted to chaotic orbits. From top to bottom: $N=10^{4.5}, N=10^{5.0}, N=10^{5.5}$, and $N=10^{6.0}$.

potential fails and orbits in frozen- $N$ systems are very different from smooth-potential characteristics. In particular, the usual distinctions between regularity and chaos that exist in a smooth potential seem completely lost. However, for larger $N$ the distinctions become clearer, and they are manifest in the evolution of "regular" versus "chaotic" clumps of initially localized particles. Just as for clumps evolved in a smooth potential, the emittance of a regular clump evolved in a frozen- $N$ potential grows as a power law in time, whereas for a chaotic clump it grows roughly exponentially. However, in both cases the growth is more rapid than in the smooth potential. Discreteness effects accelerate emittance growth for both regular and chaotic clumps. In terms of both the statistics of collections of orbits and the complexities of individual orbits, discreteness effects can be well mimicked by Gaussian white noise in the context of a Fokker-Planck/Langevin description. This appears true even when considering the short-time behavior of individual orbits. These findings suggest strongly that Langevin simulations are useful for assessing the importance of discreteness effects in real beams for which the constituent number of particles $N \rightarrow \mathcal{N}$ is too large to allow honest direct-summation integrations. Moreover, discreteness effects can remain important even if $\mathcal{N}$ is very large, e.g., in high-brightness beams, especially when chaotic orbits are present.

Discreteness effects are also important because they can trigger transitions between regular and chaotic behavior. The larger the value of $N$, the longer it takes for these transitions to become important, and they become impossible in the continuum limit. However, for any finite $N$ there appears to be a finite time beyond which it is unsafe to ignore these discreteness-induced transitions. Even if discreteness effects were too weak to facilitate frequent transitions between regularity and chaos, they may nonetheless play an important role in accelerating diffusion through a complex chaotic phase space. Generic smooth potentials admitting both regular and chaotic orbits have phase spaces in which chaotic regions are partitioned by complex structures associated with cantori in two dimensions and the Arnold web in three dimensions. Although they are not absolute obstructions, they serve as "entropy barriers" that impede phasespace transport [32]. However, even very-low-amplitude Gaussian white noise has been shown dramatically to accelerate diffusion through such barriers [33]. Since we have shown herein that discreteness effects can be modeled as Gaussian white noise, they should act as a significant source of accelerated phase-space transport.

The meaning of "chaos" in connection with beams is somewhat subtle. We have seen that two distinct sources of chaos can exist, associated with physics on different scales. Chaos associated with close encounters between individual charges will always be present. They cause nearby orbits to diverge exponentially until a distance comparable to the interparticle spacing separates them. In that context beams are always "locally chaotic." However, if in the continuum limit the bulk potential admits global stochasticity, then the orbits will continue to separate and exponentially fill global regions of phase space. These two distinct phases are characterized separately by different sets of Lyapunov exponents. Close encounters trigger an exponential separation of nearby trajectories at a rate $\chi_{N}$, but the separation saturates at only microscopic scales. The bulk potential triggers an exponential separation at a rate $\chi_{S}$ typically much smaller than $\chi_{N}$, but it saturates on macroscopic scales. Hence, when global stochasticity is present in beams, it leads to macroscopic, operationally irreversible evolution.

All of these considerations have practical implications for beams. In particular, discreteness effects can be important in real beams and over real acceleration time scales, thereby vitiating the Vlasov-Poisson methodology. In turn, simulations that correctly account for the full scale of evolutionary mechanisms may require a huge number of macroparticles, possibly comparable to the number of particles in the real beam bunch itself.

\section{ACKNOWLEDGMENTS}

Regrettably, H. E. K. died suddenly on 18 October 2003. This paper stands as a tribute to his myriad contributions toward understanding the nonlinear dynamics of Coulomb systems. He was supported in part by NSF AST-0070809 and AST-0307351. I.V.S. and C. L. B. were supported by the Department of Education Grant No. G1A62056.

[1] R.C. Davidson, Physics of Nonneutral Plasmas (Addison-Wesley, Redwood City, 1990). 
[2] M. Reiser, Theory and Design of Charged Particle Beams (Wiley, New York, 1994).

[3] R. C. Davidson, Phys. Rev. Lett. 81, 991 (1998).

[4] H. Qin, R. C. Davidson, and W.W. Lee, Phys. Rev. ST Accel. Beams 3, 084401 (2000).

[5] J. Struckmeier, Phys. Rev. E 54, 830 (1996).

[6] This order-of-magnitude estimate follows straightforwardly by, for example, combining Eqs. (5.247), (6.154), and (6.155) from M. Reiser's book, Theory and Design of Charged Particle Beams (Ref. [2]).

[7] R. Balescu, Statistical Dynamics: Matter Out of Equilibrium (Imperial College Press, London, 1997).

[8] H. E. Kandrup and I.V. Sideris, Phys. Rev. E 64, 056209 (2001).

[9] I.V. Sideris and H. E. Kandrup, Phys. Rev. E 65, 066203 (2002).

[10] C. L. Bohn and I. V. Sideris, Phys. Rev. ST Accel. Beams 6, 034203 (2003).

[11] M. N. Rosenbluth, W. M. MacDonald, and D. L. Judd, Phys. Rev. 107, 1 (1957).

[12] S. Chandrasekhar, Rev. Mod. Phys. 15, 1 (1943).

[13] See, e.g., N.G. van Kampen, Stochastic Processes in Physics and Chemistry (North-Holland, Amsterdam, 1981).

[14] For example, see Space Charge Dominated Beams and Applications of High-Brightness Beams, edited by S.Y. Lee, AIP Conf. Proc. No. 377 (AIP, New York, 1996).

[15] W. H. Press, S.A. Teukolsky, W.T. Vetterling, and B. P. Flannery, Numerical Recipes in C (Cambridge University Press, New York, 1993).

[16] G. Bennetin, L. Galgani, A. Giorgilli, and J. M. Strelcyn, Meccanica 15, 9 (1980)
[17] H. E. Kandrup, B. L. Eckstein, and B. O. Bradley, Astron. Astrophys. 320, 65 (1997).

[18] R. H. Miller, Astrophys. J. 140, 250 (1964).

[19] S. Chandrasekhar, Ellipsoidal Figures of Equilibrium (Yale, New Haven, 1969).

[20] R.W. Hockney and J.W. Eastwood, Computer Simulations Using Particles (Hilger, Philadelphia, 1988).

[21] F. J. Sacherer, in Proceedings of the 1971 Particle Accelerator Conference (IEEE, Piscataway, NJ, 1971), p. 1106.

[22] H. E. Kandrup and I.V. Sideris, Celestial Mech. 82, 61 (2002).

[23] C. L. Bohn, Phys. Rev. Lett. 70, 932 (1993).

[24] J. Struckmeier, Phys. Rev. ST Accel. Beams 3, 034202 (2000), cf. Eqs. (34) and (35).

[25] B. Chirikov, Phys. Rep. 52, 263 (1979).

[26] J. J. Tennyson, in Nonlinear Dynamics and the BeamBeam Interactions, edited by M. Month and J. C. Herrera (AIP, New York, 1979).

[27] K. Rauch and S. Tremaine, New Astron. 1, 149 (1996).

[28] M. Brown and M. Reiser, Phys. Plasmas 2, 965 (1995).

[29] M. Reiser and N. Brown, Phys. Rev. Lett. 74, 1111 (1995).

[30] See, e.g., C. L. Bohn and J. R. Delayen, Phys. Rev. E 50, 1516 (1994).

[31] H. E. Kandrup, I. M. Vass, and I. V. Sideris, Mon. Not. R. Astron. Soc. 341, 927 (2003).

[32] A. J. Lichtenberg and M. A. Lieberman, Regular and Chaotic Dynamics (Springer, Berlin, 1992).

[33] I. V. Pogorelov and H. E. Kandrup, Phys. Rev. E 60, 1567 (1999). 